\title{
Ameliorative Effect of Parishin C Against Cerebral Ischemia-Induced Brain Tissue Injury by Reducing Oxidative Stress and Inflammatory Responses in Rat Model
}

\author{
Tao Wang ${ }^{1} *$ \\ Haibo Chen ${ }^{2, *}$ \\ Shuyun $\mathrm{Xia}^{3}$ \\ Xiaofang Chen' \\ Hu Sun ${ }^{1}$ \\ Zhixin Xu (D)
}

'Department of Anesthesiology, the Second Affiliated Hospital of Hainan Medical University, Haikou, Hainan, 5703 II, People's Republic of China; ${ }^{2}$ Department of Blood Transfusion, the Second Affiliated Hospital of Hainan Medical University, Haikou, Hainan,

5703 I I, People's Republic of China; ${ }^{3}$ Department of Respiratory Medicine, Pingdu People's Hospital, Pingdu, Shandong, 266700, People's Republic of China

*These authors contributed equally to this work
Correspondence: Zhixin Xu

The Second Affiliated Hospital of Hainan Medical University, 368 Yehai Av, Haikou, Hainan, 5703II, People's Republic of China

Email xuzhix201I@I63.com
Background: Gastrodia elata Blume (Orchidaceae) is a widely used traditional Chinese herbal medicine in the clinical practice of China, to treat nervous headache, convulsions, dizziness, neurasthenia, and so on. Parishin C (Par C), one of the major bioactive components of Gastrodia elata Blume, is known to exert many different biological activities, including antipsychotic and neuroprotective effects. However, there is little research about its neuroprotective effect in an ischemic stroke model. The objective of the present study is thus to investigate the neuroprotective effects of Par $\mathrm{C}$ against cerebral ischemia damage.

Methods: Rats were pretreated with Par C (25, 50, or $100 \mathrm{mg} / \mathrm{kg} /$ day, i.p.) for 21 days, then subjected to $2 \mathrm{~h}$ of middle cerebral artery occlusion (MCAO) and $22 \mathrm{~h}$ of reperfusion. Neurological deficient scores, brain water content, histopathology, TCC staining were performed to assess the neuroprotective effects of Par C. Meanwhile, the oxidative stress, inflammation and apoptosis-related markers of brain tissue were evaluated by corresponding assay kits. Besides, the antioxidant and pro-inflammatory expression was measured by realtime quantification PCR (RT-qPCR).

Results: Our findings indicated that the pre-treatment with Par $\mathrm{C}$ improved nerve function, suppressed oxidative stress, and pro-inflammatory factors release in rats with cerebral ischemia damage. Besides, Par C significantly increased antioxidant expression and declined pro-inflammatory cytokines expression.

Conclusion: Par C is shown to exert neuroprotective effects partly via inhibiting oxidative stress and inflammation in a rat model of MCAO.

Keywords: antioxidant, inflammation, brain damage, stroke, parishins

\section{Introduction}

Cerebral stroke, including hemorrhagic and ischemic stroke, is one of the primary causes of mortality and disability worldwide, which also brings an economic burden to countries and families. ${ }^{1}$ In all cerebral stroke cases worldwide, ischemic stroke is the most common type of stroke and is evoked due to insufficient blood supply or blockage of blood vessels in the brain tissue, resulting in brain injury and neurological impairment. ${ }^{2}$ Currently, drug therapy, stent implantation, and thrombolysis are the primary treatment for cerebral stroke. ${ }^{3}$ Because of the adverse reactions of long-term drug use, unstable safety, and limited therapeutic time window, these therapies have drawbacks in the clinical treatment. Therefore, there is an urgent need for the development of effective therapeutic drugs with lower side effects. 
Although the pathological mechanisms of cerebral ischemia damage are multifactorial and complex, it has been reported that inflammation, oxidative stress, acidosis, and excitotoxicity are the primary fundamental mechanisms involved in the pathology of ischemia stroke. ${ }^{4}$ It has been reported that cellular inflammatory responses play an important role in the pathogenesis of cerebral ischemia. ${ }^{5}$ Cerebral ischemia could decrease oxygen and glucose supply, further disturb the normal energetics of nerve cells, which resulted in mitochondrial oxidative stress. ${ }^{6}$ Additionally, oxidative stress could evoke the generation of NF- $\mathrm{kB}$ that increase the secretion of inflammatory cytokines. ${ }^{7}$ These series of events trigger further aggravate the formation of cerebral ischemia. Therefore, agents that could exert anti-inflammatory, and anti-oxidant properties are helpful to alleviate cerebral ischemia injury. ${ }^{8}$

Traditional Chinese Medicines are rich in bioactive ingredients for the development of conventional medicines. Gastrodia elata Blume (Orchidaceae) is a wellknown traditional Chinese herbal medicine that has long been used to treat nervous headache, convulsions, dizziness, neurasthenia, and other neurological disorders. ${ }^{9}$ It has been reported that parishins could improve animal performances in various cognitive-behavioral tests. ${ }^{9}$ Parishin C (Par C) is a bioactive compound isolated from the Gastrodia elata Blume and has been reported to exert various pharmacological activities, including antipsychotic and neuroprotective effects. A previous study showed that Par C improved schizophrenia-like psychosis via activation of 5-hydroxytryptamine $1 \mathrm{~A}\left(5-\mathrm{HT}_{1 \mathrm{~A}}\right)$ receptors. ${ }^{10} \mathrm{Par}$ $\mathrm{C}$ showed protection effects against $\mathrm{A} \beta$-induced long-term potentiation damage via N-methyl-D-aspartate (NMDA) receptors. ${ }^{11}$ Besides, Parishin could improve the activity of SOD, and decrease MDA and ROS levels of yeast under oxidative stress conditions. ${ }^{12}$ However, the neuroprotective effect of Par $\mathrm{C}$ on cerebral ischemia damage has not been investigated. Therefore, the present study aimed to investigate the neuroprotective effects of Par on cerebral ischemia injury in rats.

\section{Materials and Methods}

\section{Experimental Animals}

Male Wistar rats (8-10 weeks old and weighing 200-220 g) were procured from the Experimental Animal Center of Hainan Province. All animals were maintained under a standard animal room with a natural $12 / 12 \mathrm{~h}$ dark-light cycle (humidity $55 \%-65 \%$ and temperature $20-24^{\circ} \mathrm{C}$ ). All the animal experiments were approved by the Animal Research Ethics Committee of The Second Affiliated Hospital of Hainan Medical University (Approval number: 20200081) and carried out based on the National Standard (GB/T 35892-2018) for Laboratory Animals Care and Use.

\section{Animal Grouping and Experimental Protocol}

Edaravone (Eda, $3 \mathrm{mg} / \mathrm{kg}$ ) was shown to alleviate cerebral ischemia damage in rats, so it used as a positive drug in the present study. ${ }^{13}$ Rats were randomly divided into six groups (each group containing 16 rats) as follows: Sham group, rats were intraperitoneally injected with sterile saline for three weeks; MCAO group, rats were intraperitoneally injected with sterile saline for three weeks prior to MCAO and subjected to $2 \mathrm{~h}$ of MCAO followed by 22 $\mathrm{h}$ of reperfusion; $\mathrm{MCAO}+\mathrm{LPar}$, rats were intraperitoneally injected with low dose Par C ( $25 \mathrm{mg} / \mathrm{kg} /$ day $)$ for three weeks prior to MCAO and subjected to $2 \mathrm{~h}$ of MCAO followed by $22 \mathrm{~h}$ of reperfusion; MCAO+MPar, rats were intraperitoneally injected with medium dose Par C (50 mg/ $\mathrm{kg} / \mathrm{day}$ ) for three weeks prior to MCAO and subjected to 2 $\mathrm{h}$ of MCAO followed by $22 \mathrm{~h}$ of reperfusion; MCAO +HPar, rats were intraperitoneally injected with high dose Par C $(100 \mathrm{mg} / \mathrm{kg} /$ day $)$ for three weeks prior to MCAO and subjected to $2 \mathrm{~h}$ of MCAO followed by 22 $\mathrm{h}$ of reperfusion; MCAO+Eda, rats were intraperitoneally injected with Eda (3 $\mathrm{mg} / \mathrm{kg} /$ day) for three weeks prior to MCAO and subjected to $2 \mathrm{~h}$ of MCAO followed by 22 $\mathrm{h}$ of reperfusion. ${ }^{13}$ Sham group rats performed the same surgical procedure, except MCAO. Establishment of MACO model was referenced on previous report. ${ }^{14} \mathrm{Par}$ $\mathrm{C}$ and Eda were purchased from Sigma-Aldrich (purity $>$ 98\%) and dissolved in saline and freshly prepared before use. The dose of Par C was based on previous literature. ${ }^{10}$ Later, a rat model of MCAO was established for the induction of cerebral ischemia. All rats were anesthetized by intraperitoneal injection of xylazine $(5 \mathrm{mg} / \mathrm{kg})$ and ketamine $(45 \mathrm{mg} / \mathrm{kg})$ mixture, the dose xylazine and ketamine was based on previous report. ${ }^{15}$ The body temperature was maintained at $36-38^{\circ} \mathrm{C}$, while the surgical procedure was performed. A midline incision was performed and the right common carotid artery was exposed. Then, a monofilament nylon thread with a heat-rounded tip was used to occlude the middle cerebral artery. The sham group rats were subjected to a similar surgical procedure 
without cerebral artery occlusion. After $2 \mathrm{~h}$ MCAO and 22 $\mathrm{h}$ reperfusion, all rats were assessed for behavioral deficits.

\section{Neurological Deficit Scores}

After $2 \mathrm{~h} \mathrm{MCAO}$ and $22 \mathrm{~h}$ reperfusion, the neurological deficit scores were assessed by a blinded investigator according to previous report. ${ }^{3}$ Neurological deficit scores were scored on a 4-point scale as follows: 0, no neurological deficit; 1, failure to fully extend the forepaw; 2, circling to the opposite side when walking; 3 , falling to the opposite side; and 4, had depression and could not walk spontaneously.

\section{Brain Water Content}

After the rats were anesthetized, the brain tissues were removed quickly and weighted to record wet weight. Then, the brain tissues were dried at $95^{\circ} \mathrm{C}$ for $48 \mathrm{~h}$ and weighed again to record the dry weight. The water content of brain tissue was calculated using the following formula:

Brain water content $(\%)=($ wet weight-dry weight $) /$ wet weight $\times 100$

\section{Measurement of Infarct Volume}

Brain samples were cut into $2 \mathrm{~mm}$ thick sections. The sections were stained with 2,3,5-triphenyltetrazolium chloride TTC $(2 \%)$ at $37^{\circ} \mathrm{C}$ for $0.5 \mathrm{~h}$ and fixed with $4 \%$ paraformaldehyde at $4^{\circ} \mathrm{C}$ overnight. Sections were photographed and the size of cerebral infarction was measured and analyzed with Image $\mathrm{J}$.

\section{Histopathology Analysis of Brain Tissue}

After $2 \mathrm{~h} \mathrm{MCAO}$ and $22 \mathrm{~h}$ reperfusion, the rats were anesthetized and cardiac perfused with $0.9 \% \mathrm{NaCl}$. Then, the brain tissues were removed quickly and postfixed in $4 \%$ paraformaldehyde overnight, and embedded in paraffin. The $5 \mu \mathrm{m}$ thick sections were cut and stained with hematoxylin and eosin. The histopathology changes were observed under an optical microscope (Olympus BX50, Japan).

\section{Preparation of Tissue Homogenate}

After $2 \mathrm{~h}$ MCAO and $22 \mathrm{~h}$ reperfusion, the rats were anesthetized and brain tissues were collected quickly. After the brain tissues were washed with ice-cold phosphate buffer saline, brain tissues were homogenized in 9 volumes of ice-cold phosphate buffer saline. Then, the brain tissue homogenate was centrifuged at $10,000 \mathrm{~g}\left(4^{\circ} \mathrm{C}\right)$ for $10 \mathrm{~min}$. The supernatant was collected for biochemical analysis.
The total protein content of tissue homogenate was measured using commercially available kits (Nanjing Jiancheng Bioengineering Institute, Nanjing, China) following the manufacturer's protocols.

\section{Estimation of Oxidative Stress, Inflammatory Markers and Apoptosis Markers}

The activities of Superoxide Dismutase (SOD), Catalase (CAT) and Glutathione peroxidase (GSH-Px), and the levels of malondialdehyde (MDA), tumour necrosis factor alpha (TNF- $\alpha$ ), Interleukin 6 (IL-6), Interleukin-1beta (IL$1 \beta)$, Caspase-3, Caspase-9, and brain-derived neurotrophic factor (BDNF) in the brain homogenate were measured using a commercially available kit (Nanjing Jiancheng Bioengineering Institute, Nanjing, China) following the manufacturer's protocols. The ELISA kit of BDNF was purchased from Shanghai Enzyme-linked Biotechnology Co., Ltd. The catalog number of the corresponding kits was as follows: SOD (A001-3-2), CAT (A007-1-1), GSHPx (A005-1-2), MDA (A003-1-2), TNF- $\alpha$ (H052), IL-6 (H007), and IL-1 $\beta$ (H002), Caspase-3 (G015-1-3), Caspase-9 (G018-1-3), BDNF (ML302829).

\section{Quantification of Oxidative Stress and Inflammatory Gene Expression}

Total RNA was extracted from brain tissue using TRIzol reagent (Invitrogen, CA, USA) following the manufacturer's protocols. Then, $2 \mu \mathrm{g}$ of RNA was converted to cDNA using PrimeScript $^{\mathrm{TM}}$ II Strand cDNA Synthesis Kit (Thermo, USA). RT-qPCR amplification was performed using $1 \mu \mathrm{L}$ of cDNA template, $10 \mu \mathrm{L}$ of SYBR advantage qPCR Master Mix kit (Thermo, USA), and $1 \mu \mathrm{L}$ of each of primer following the manufacturer's protocol. The reaction was performed as follows: $95^{\circ} \mathrm{C}$ for $1 \mathrm{~min} ; 40$ cycles of $95^{\circ} \mathrm{C}$ for $15 \mathrm{~s}, 60^{\circ} \mathrm{C}$ for $30 \mathrm{~s}$ and $72^{\circ} \mathrm{C}$ for $25 \mathrm{~s}$. Primers for RT-qPCR were shown in Table 1. The expression of target gene transcripts was normalized by the reference gene GAPDH.

\section{Statistical Analysis}

The results were shown as mean \pm S.D. The significant differences between individual groups were analyzed using one-way analysis of variance (ANOVA), followed by Tukey's test. The statistical analysis was carried out by using GraphPad Prism 7.0 (Graphpad Software Inc., CA, USA). $P<0.05$ was considered statistically significant. 
Table I Primers for Quantitative Real-Time PCR

\begin{tabular}{|l|l|l|}
\hline Gene & Forward Primer & Reverse Primer \\
\hline SODI & AGGTCGGTGTGAACGGATTTG & GGGGTCGTTGATGGCAACA \\
CAT & CCTATTGCCGTTCGATTCTC & CCCACAAGATCCCAGTTACC \\
GSH-Px & CCAGGAGAATGGCAAGAATG & AAGGTAAAGAGCGGGTGAGC \\
TNF- $\alpha$ & TCAGCCGATTTGCTATCTCATA & AGTACTTGGGCAGATTGACCTC \\
IL-6 & TGGTGATAAATCCCGATGAAG & GGCACTGAAACTCCTGGTCT \\
IL-I $\beta$ & TGAAATGCCACCTTTTGACAG & CCACAGCCACAATGAGTGATAC \\
NF-KB & AGCACCAAGACCGAAGCAA & TCTCCCGTA ACCGCGTAGTC \\
GAPDH & AGGTCATCCCAGAGCTGAACG & CACCCTGTTGCTGTAGCCGTAT \\
\hline
\end{tabular}

\section{Results}

\section{Neuroprotective Effects of Parishin C in Rats with Cerebral MCAO Damage}

To explore the neuroprotective effects of Par on brain damage in MCAO rats, we first assessed the neurological scores and brain water content. As shown in Figure 1A, the MCAO group rat showed an increased level of neurological deficit scores compared to the Sham group $(P<0.001)$. The neurological deficit scores was significantly decreased after Eda or Par $\mathrm{C}(25,50$ and $100 \mathrm{mg} / \mathrm{kg} /$ day $)$ pretreatment in a dosedependent manner. The changing trend of the brain water content was similar to that of neurological scores (Figure 1B). The brain water content of MCAO rats was increased compared to the Sham group $(P<$ 0.001). In the MCAO and LPar groups, there were no significant changes on brain water content. The brain water content was significantly decreased after Eda or Par C (50 and $100 \mathrm{mg} / \mathrm{kg} /$ day) pretreatment in a dosedependent manner. There were no significant differences in brain water content and neurological deficit scores between the HPar and Eda groups $(P>0.05)$. These findings indicated that Par $\mathrm{C}$ pretreatment could alleviate cerebral infarction in a rat model.
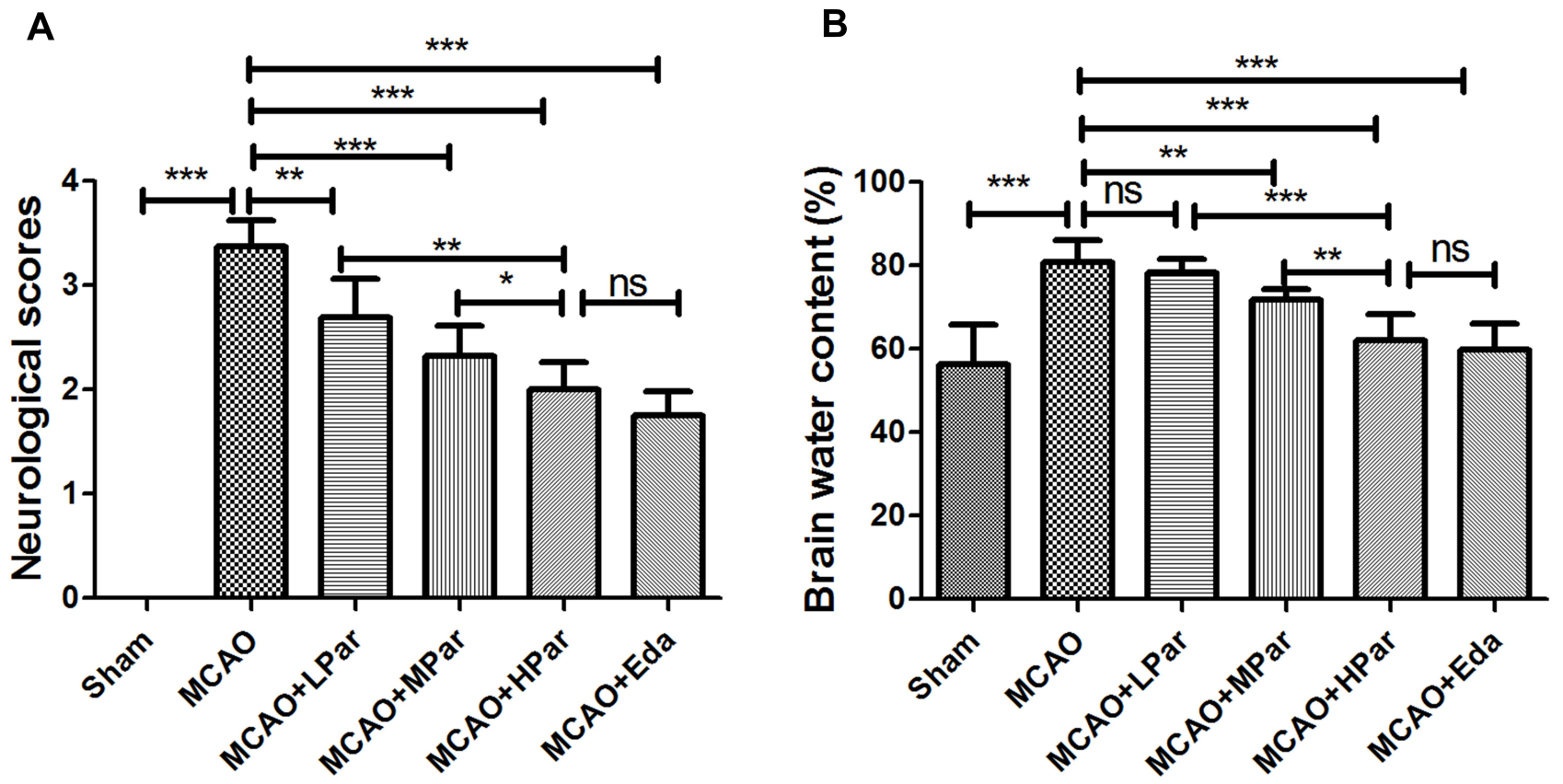

Figure I Neuroprotective effects of Par C in rats subjected to MCAO damage. Neurological deficit score $(\mathbf{A})$ and brain water content (B) in different groups. Data were shown as mean $\pm S D(n=8)$. $* * * P<0.001$, **P < 0.01, *P<0.05, no significant difference (ns). MCAO+LPar: 25 mg/kg body weight Par $C$ treatment dose; MCAO+MPar: $50 \mathrm{mg} / \mathrm{kg}$ body weight Par C treatment dose; MCAO+HPar: $100 \mathrm{mg} / \mathrm{kg}$ body weight Par $\mathrm{C}$ treatment dose; MCAO+Eda: $3 \mathrm{mg} / \mathrm{kg}$ body weight Eda treatment dose. Abbreviations: SD, standard deviation; Par C, Parishin C; MCAO, middle cerebral artery occlusion. 
Sham

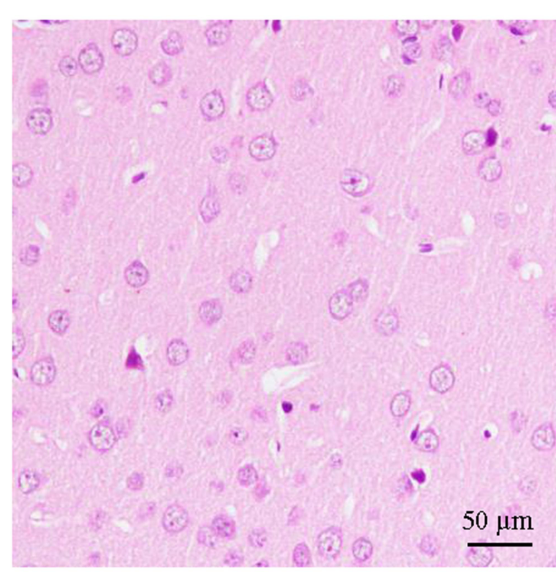

MCAO+MPar

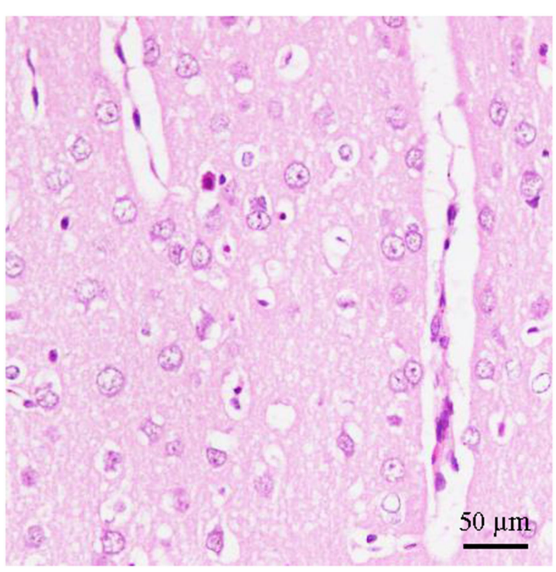

MCAO

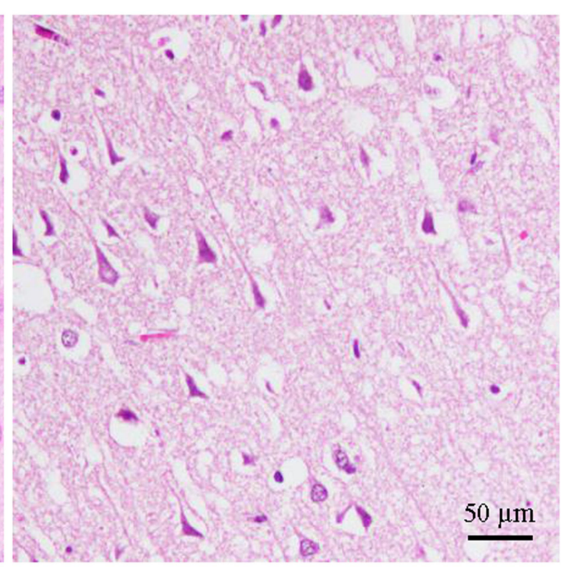

MCAO+LPar

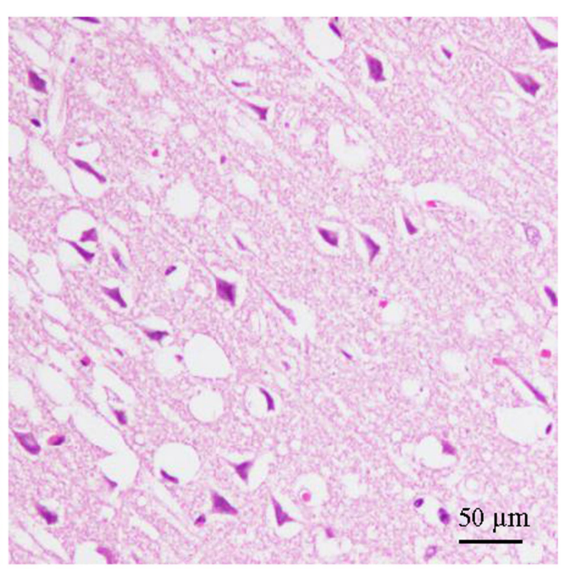

MCAO+HPar

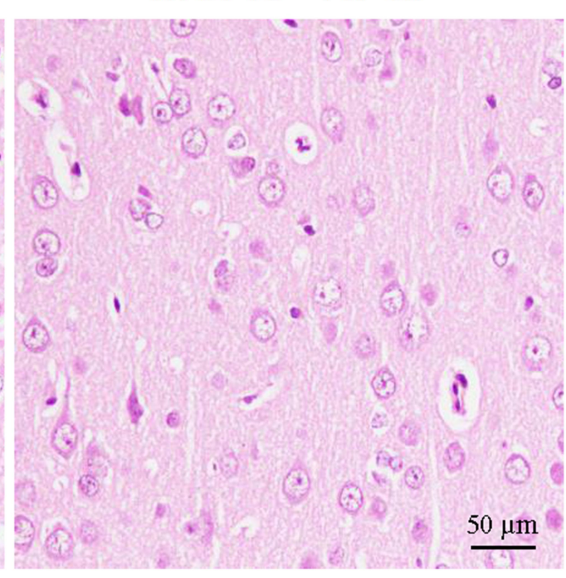

$\mathrm{MCAO}+\mathrm{Eda}$

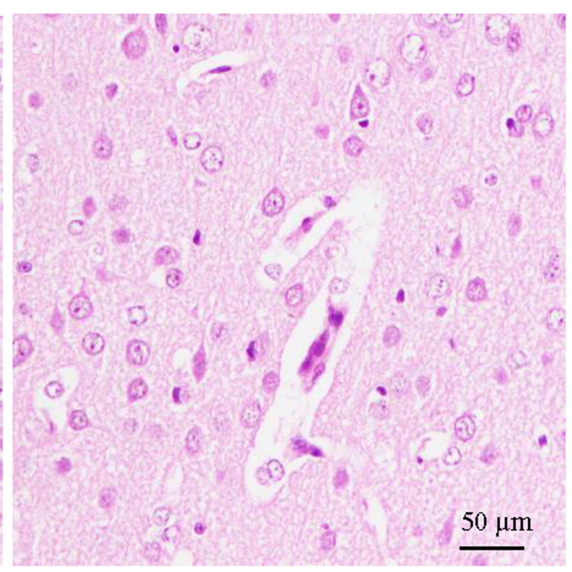

Figure 2 Effect of Par C on histopathological change of the brain tissue after MCAO. Scale bar $=50 \mu$ m, with magnification $200 \times$. The sham group exhibited the normal morphologic features of neurons. The MCAO group exhibited the presence of vacuolated interspaces and disordered brain neuronal arrangement. Par $C$ and Eda alleviated the injury of neurons in the MCAO group. MCAO+LPar: $25 \mathrm{mg} / \mathrm{kg}$ body weight Par C treatment dose; MCAO+MPar: $50 \mathrm{mg} / \mathrm{kg}$ body weight Par C treatment dose; MCAO +HPar: $100 \mathrm{mg} / \mathrm{kg}$ body weight Par treatment dose; MCAO+Eda: $3 \mathrm{mg} / \mathrm{kg}$ body weight Eda treatment dose.

Abbreviations: Par C, Parishin C; MCAO, middle cerebral artery occlusion; Eda: edaravone.

\section{Histopathological Analysis of Brain}

To further confirm the neuroprotective effects of Par $\mathrm{C}$ on cerebral MCAO damage, HE staining was carried out on the brain tissues. As shown in Figure 2 and Table 2, the sham group exhibited intact neurons and no lesion. However, the MCAO group showed the presence of vacuolated interspaces and disordered brain neuronal arrangement. Besides, neuronal necrobiosis and inflammatory cell infiltration were obviously observed in MCAO group. These histopathology changes of cerebral MCAO damage in $\mathrm{MCAO}+\mathrm{MPar}, \mathrm{MCAO}+\mathrm{HPar}$, and MCAO+Eda groups were improved to varying degrees. The MCAO + HPar and MCAO+Eda groups possessed better protective effect on nerve cells than the MCAO+MPar group. Table 2 showed the semiquantitatively scores (H\&E) of brain tissues of the different groups. These results indicated that the Par $\mathrm{C}$ exerted protection effects against MCAOinduced histopathological changes in the brain.

Table 2 Hisopathologic Findings (H\&E) of Brain Tissues of the Different Groups

\begin{tabular}{|l|c|c|c|c|c|c|}
\hline & Sham & MCAO & MCAO+LPar & MCAO+MPar & MCAO+HPar & MCAO+Eda \\
\hline Neuronal necrobiosis & - & +++ & +++ & ++ & + & + \\
Inflammatory cell infiltration & - & +++ & +++ & ++ & + \\
\hline
\end{tabular}

Notes: H\&E stained sections semiquantitatively scored as no lesion (-), mild (+), moderate $(++)$, and severe $\left(^{+++}\right)$. 


\section{Alleviation of Oxidative Stress Evoked by MCAO Damage in Brain by Par \\ C Pretreatment}

As shown in Figure 3, MCAO damage induced oxidative stress via depletion of endogenous antioxidants in the brain tissue. The activities of SOD, Catalase, GSH-Px in brain tissue of the MCAO rats were declined compared with the Sham group $(P<0.001)$. In the MCAO and LPar groups, there were no significant changes on antioxidant enzyme activities and MDA content $(P>0.05)$. However, the antioxidant enzyme activities were significantly increased after Eda or Par C (50 and $100 \mathrm{mg} / \mathrm{kg} /$ day) pretreatment in a dose-dependent manner. There were no significant differences in antioxidant enzyme activities between the HPar and Eda groups $(P>0.05)$. The content of MDA in brain tissue of the MCAO rats was increased
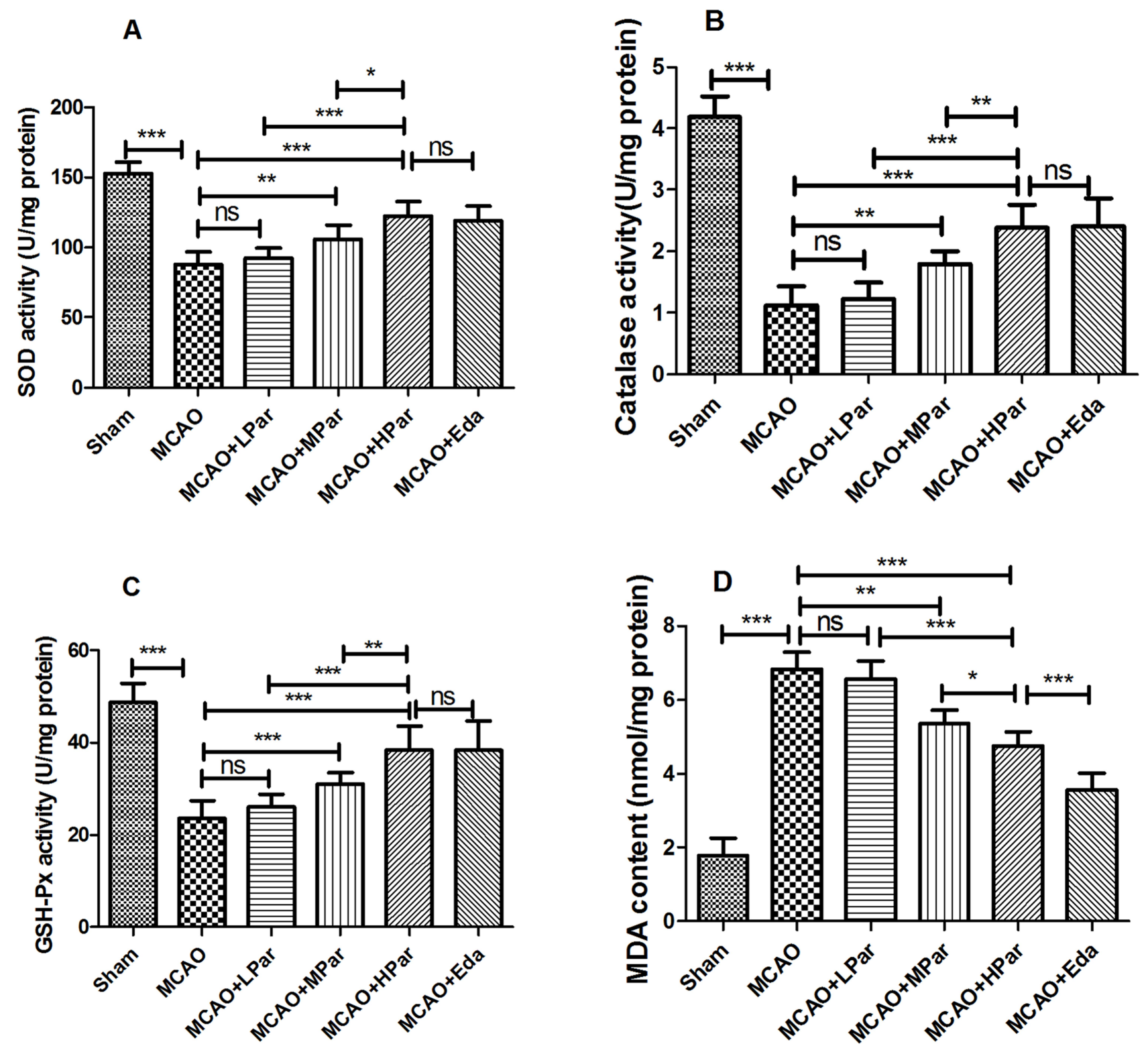

Figure 3 Effect of Par C on oxidative stress markers in the brain tissue after MCAO. SOD activity (A), catalase activity (B), GSH-Px activity (C), and MDA content (D) in brain tissue were measured by ELISA. Data were shown as mean $\pm \mathrm{SD}(\mathrm{n}=8)$. ***P $<0.00 \mathrm{l}$, **P $<0.0 \mathrm{l}$, *P< 0.05 , no significant difference (ns). MCAO+LPar: $25 \mathrm{mg} / \mathrm{kg}$ body weight Par treatment dose; MCAO+MPar: $50 \mathrm{mg} / \mathrm{kg}$ body weight Par C treatment dose; MCAO+HPar: $100 \mathrm{mg} / \mathrm{kg}$ body weight Par C treatment dose; MCAO+Eda: $3 \mathrm{mg} / \mathrm{kg}$ body weight Eda treatment dose.

Abbreviations: SD, standard deviation; Par C, Parishin C; MCAO, middle cerebral artery occlusion; SOD, superoxide dismutase; GSH-Px, glutathione peroxidase; MDA, malondialdehyde. 
compared with the Sham group $(P<0.001)$. However, the MDA level was significantly decreased after Eda or Par $\mathrm{C}(25,50$ and $100 \mathrm{mg} / \mathrm{kg} /$ day $)$ pretreatment in a dosedependent manner.

\section{Par C Improved the Antioxidant Enzymes Gene Expression in the Brain Tissue After MCAO}

As shown in Figure 4, the mRNA expression levels of SOD1, CAT, and GSH-Px in the MCAO group were lower than those in the Sham group $(P<0.001)$. After treatment with Par C $(100 \mathrm{mg} / \mathrm{kg})$ or Eda, SOD1, CAT, and GSH-Px expression levels were up-regulated. There were no significant differences in antioxidant enzyme expression levels between the HPar and Eda groups $(P>0.05)$. These findings implied that Par $\mathrm{C}$ could suppress oxidative stress induced by MCAO injury in rats.

\section{Amelioration of Inflammation Induced by MCAO Damage in Brain by Par \\ C Pretreatment}

As shown in Figures 5 and 6, the levels of TNF- $\alpha$, IL-6, IL-1 $\beta$, and NF- $\mathrm{KB}$ in the MCAO group were higher than those in the Sham group $(P<0.001)$. After treatment with Par C (100 mg/kg) or Eda, TNF- $\alpha$, IL-6, IL-1 $\beta$, and NF- $\kappa B$ levels were decreased. These findings implied that Par $\mathrm{C}$ could suppress inflammation induced by MCAO injury in rats.

\section{Effects of Par C on the Activities of Apoptosis} Markers and the Levels of BDNF

As shown in Figure 7A and B, the activities of Caspase-3 and Caspase-9 were increased compared with that of Sham group $(P<0.001)$. However, the activities of Caspase-3 and Caspase- 9 were decreased after Par C $(100 \mathrm{mg} / \mathrm{kg})$ or Eda pretreatment. Besides, the levels of BDNF were decreased compared with that of Sham group $(P<$ 0.001), the levels of BDNF were increased after Par C $(100 \mathrm{mg} / \mathrm{kg})$ or Eda pretreatment (Figure $7 \mathrm{C})$. There were no significant differences in apoptosis markers and the BDNF between the HPar and Eda groups $(P>0.05)$.

\section{Par C Reduced Cerebral Infarction After MCAO in Rats}

As shown in Figure 8, the infarct volume was increased compared with that of Sham group $(P<0.001)$. However, the infarct volume was decreased after Par C $(100 \mathrm{mg} / \mathrm{kg})$ or Eda pretreatment. There was no significant difference between the HPar and Eda groups $(P>0.05)$.

\section{Correlation Analysis Was Performed Between the Neurological Score and Inflammatory and Oxidative Stress \\ Parameters}

As shown in Table 3, the neurological score was positively correlated with each of hepatic TNF- $\alpha$ level $(0.9705, P<$ 0.0001), IL-6 level (0.9614, $P<0.0001)$, IL-1 $\beta$ level (0.941, $P<0.0001)$, and MDA content $(0.9668, P<$ $0.0001)$, and negatively correlated with each of hepatic SOD activity $(-0.9289, P<0.0001)$, CAT activity
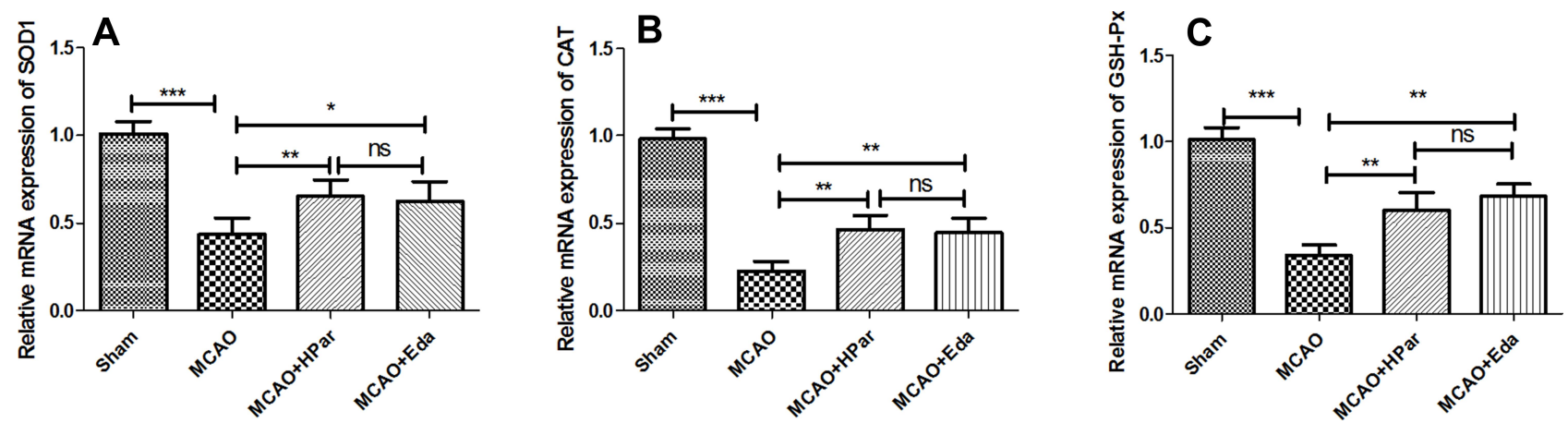

Figure 4 Effect of Par C on antioxidant gene expression in the brain tissue after MCAO. The mRNA expression levels of SODI (A), CAT (B), and GSH-Px (C) in brain tissue were measured by RT-qPCR. Data were shown as mean $\pm S D(n=6)$. $* * * P<0.001$, **P $<0.01$, ${ }^{* P}<0.05$, no significant difference (ns). MCAO+LPar: $25 \mathrm{mg} / \mathrm{kg}$ body weight Par C treatment dose; MCAO+MPar: $50 \mathrm{mg} / \mathrm{kg}$ body weight Par C treatment dose; MCAO+HPar: $100 \mathrm{mg} / \mathrm{kg}$ body weight Par C treatment dose; MCAO+Eda: $3 \mathrm{mg} /$ $\mathrm{kg}$ body weight Eda treatment dose.

Abbreviations: SD, standard deviation; Par C, Parishin C; MCAO, middle cerebral artery occlusion; SODI, copper/zinc superoxide dismutase I; CAT, catalase; GSH-Px, glutathione peroxidase. 

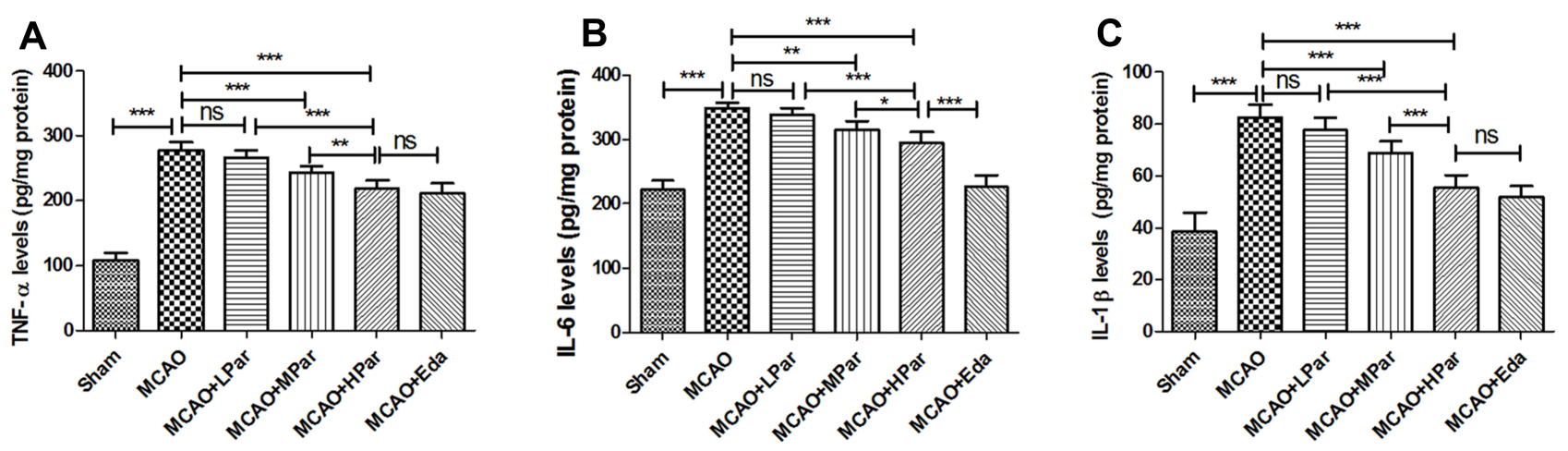

Figure 5 Par C inhibited the secretion of the pro-inflammatory cytokine in the brain tissue after MCAO. TNF- $\alpha$ (A), IL-6 (B), and IL-I $\beta$ (C) levels in brain tissue were measured by ELISA. Data were shown as mean $\pm \mathrm{SD}(\mathrm{n}=8)$. $* * * P<0.00 \mathrm{I}, * * P<0.0 \mathrm{I}, * P<0.05$, no significant difference (ns). MCAO+LPar: $25 \mathrm{mg} / \mathrm{kg}$ body weight Par $\mathrm{C}$ treatment dose; MCAO+MPar: $50 \mathrm{mg} / \mathrm{kg}$ body weight Par $\mathrm{C}$ treatment dose; MCAO+HPar: $100 \mathrm{mg} / \mathrm{kg}$ body weight Par treatment dose; MCAO+Eda: $3 \mathrm{mg} / \mathrm{kg}$ body weight Eda treatment dose.

Abbreviations: SD, standard deviation; Par C, Parishin C; MCAO, middle cerebral artery occlusion; TNF- $\alpha$, tumor necrosis factor- $\alpha$; IL-6, interleukin-6; IL-I $\beta$, interleukinI $\beta$.

$(-0.9607, P<0.0001)$, and GSH-Px activity $(-0.9037, P<$ $0.0001)$. These findings indicated that Par exerted neuroprotective effects partly via inhibiting oxidative stress, and inflammation.

\section{Discussion}

MCAO simulates the incidence of ischemic stroke evoked by a blockage or interruption of blood flow to the brain, which results in a cascade of events including depolarization of neuronal cells, neuroinflammation, the formation of oxidative radicals, and ultimately causes neuron cell death. $^{16,17}$ Although the exact molecular mechanisms of cerebral ischemic/reperfusion injury have not been completely understood, oxidative stress injury and inflammation are the major factors that evoke severe damage to biological macromolecules, resulting in neuron cell and tissue damage. ${ }^{18}$ Previous reports showed that reactive oxygen species and free radicals are released during ischemic/reperfusion injury, which initiating lipid peroxidation and leading to oxidative stress. ${ }^{19,20}$ Besides, ischemic/reperfusion injury also induces some additional problems, including generation of inflammatory cytokines, and release of free radical which further exacerbates the progression of oxidative stress, resulting in more neuron cell and tissue injury. ${ }^{21}$ Therefore, agents with anti-oxidant and anti-inflammatory properties are recommended for the prevention and treatment of cerebral ischemia. ${ }^{22-24}$

Natural products derived from medicinal plants such as catalpol, baicalin, triptolide, and salvianolic acid, which can inhibit inflammation and oxidative damage to treat neurodegenerative diseases. ${ }^{25,26}$ Gastrodia elata Blume is a well-known traditional Chinese herbal medicine that has long been used to treat nervous headache, dizziness, neurasthenia, and other neurological disorders. Additionally, Par C is a major bioactive compound from Gastrodia elata Blume, is known to exert various pharmacological activities. Thus, we speculated Par may inhibit neuroinflammation and oxidative stress, thereby, alleviate cerebral ischemic injury. In the present study, a temporary MCAO and reperfusion rat model was established to investigate the neuroprotective effects of Par C following acute cerebral ischemic injury. The present research for the first time offered scientific proof about Par $\mathrm{C}$, as a neuroprotective drug in preventive of ischemic/reperfusion damage in MCAO rats. Our findings demonstrated that Par $\mathrm{C}$ pretreatment alleviated cerebral ischemic injury following MCAO. Par $\mathrm{C}$ could improve antioxidant enzyme activities and inhibit the generation of lipid peroxidation following cerebral ischemic injury. Moreover, our finding revealed that Par $\mathrm{C}$ could inhibit the secretion of proinflammatory cytokines in the brain tissue after MCAO.

It has been reported that cerebral ischemic/reperfusion injury could cause cerebral infarction and nerve damage. ${ }^{27}$ Neurological deficit scores, brain water content, and the degree of the lesion are the major indicators for assessing brain damage. Accumulating pieces of evidence have indicated that oxidative stress is involved in cerebral ischemic/ reperfusion injury and have been demonstrated a positive correlation between neuronal injury, generation of free radical, and lipid peroxidation. ${ }^{28,29}$ Besides, the endogenous antioxidant defense systems including CAT, GSH-Px, and SOD systems are disturbed or excess free radicals are formed during the cerebral ischemic damage, which is also accompanied by the increase of lipid peroxidation in the 

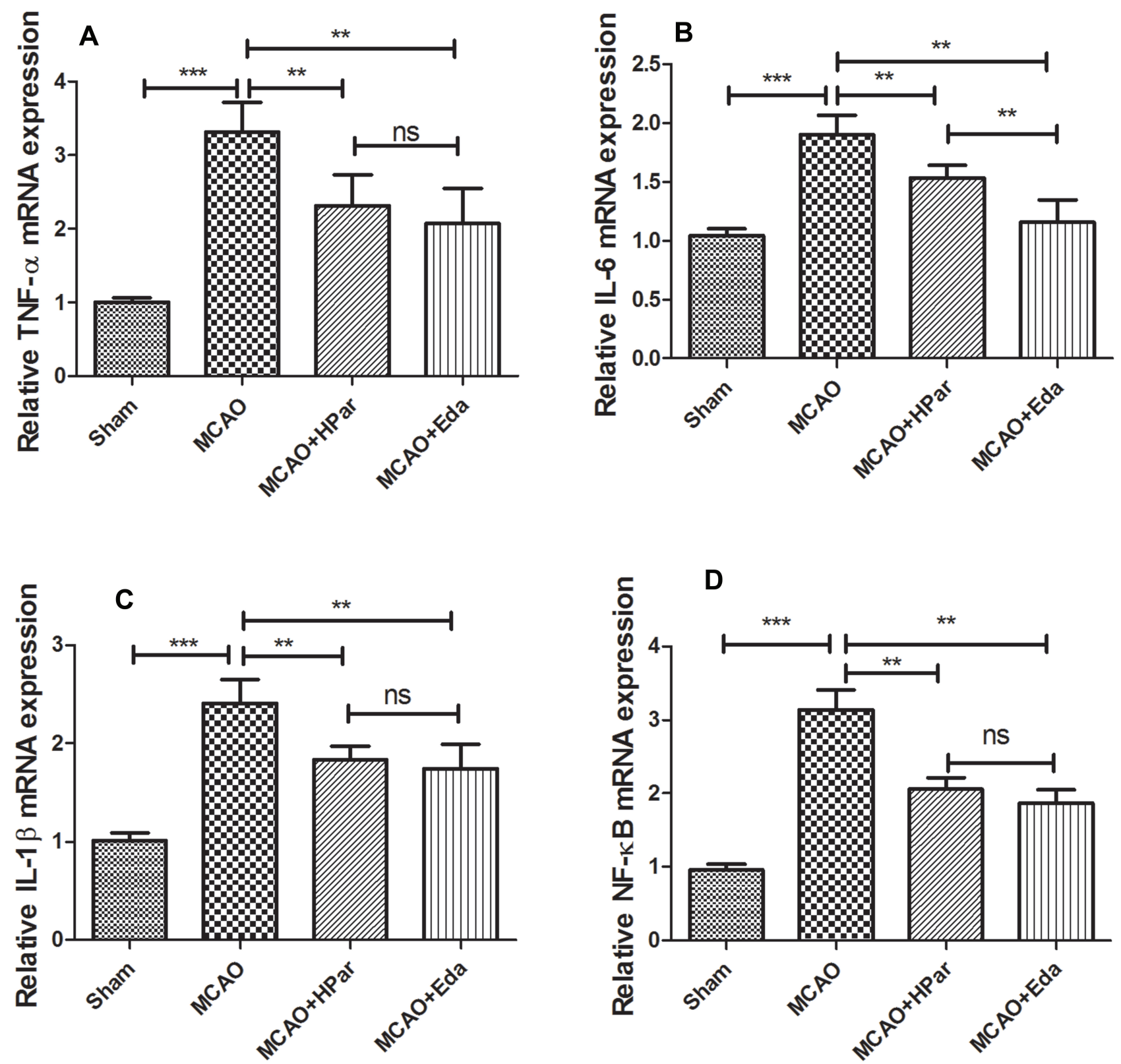

Figure 6 Par $C$ down-regulated the inflammation gene expression in the brain tissue after MCAO. The mRNA expression levels of TNF- $\alpha$ (A), IL-6 (B), IL-I $\beta$ (C), and NF$\kappa B(D)$ level in brain tissue were measured by RT-qPCR. Data were shown as mean $\pm S D(n=6)$. $* * * P<0.001$, ***P< 0.01 , no significant difference (ns). MCAO+LPar: $25 \mathrm{mg} /$ $\mathrm{kg}$ body weight Par C treatment dose; MCAO+MPar: $50 \mathrm{mg} / \mathrm{kg}$ body weight Par $\mathrm{C}$ treatment dose; MCAO+HPar: $100 \mathrm{mg} / \mathrm{kg}$ body weight Par C treatment dose; MCAO +Eda: $3 \mathrm{mg} / \mathrm{kg}$ body weight Eda treatment dose.

Abbreviations: SD, standard deviation; Par C, Parishin C; MCAO, middle cerebral artery occlusion; TNF- $\alpha$, tumor necrosis factor- $\alpha$; IL-6, interleukin-6; IL-I $\beta$, interleukin-I $\beta$.

brain tissue. ${ }^{15}$ Therefore, improvement of the antioxidant defense system, including increased the activities of CAT, GSH-Px, and SOD, and decreased MDA content may be beneficial for neuronal recovery following cerebral ischemic damage. Parishin has been demonstrated to extend the lifespan of yeast partly via inhibiting oxidative stress. $^{12}$ Consistent with our present research, MCAO induced oxidative stress of brain tissue, whereas Par
C pretreatment improved the activities of CAT, GSH-Px, and SOD, and decreased MDA level in MCAO rats. The excessive generation of reactive oxygen species also evokes neuronal inflammation. More and more people have realized the importance of continuous inflammatory processes after ischemia including recruiting blood inflammatory cells, releasing pro-inflammatory factors, and activating microglia cells. ${ }^{30,31}$ An increasing amount of 

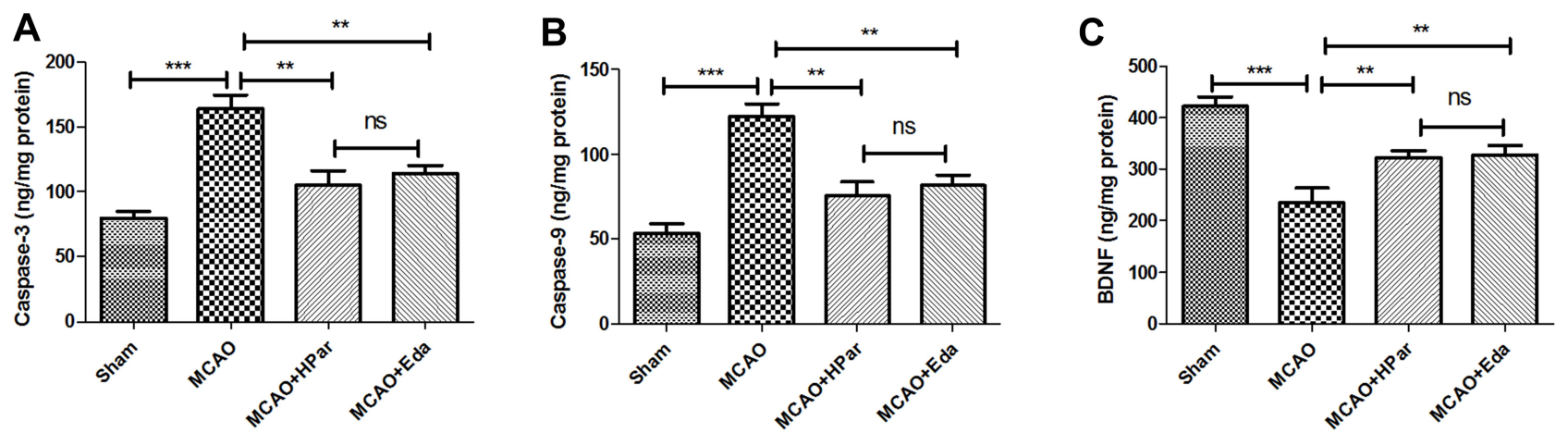

Figure 7 Effects of Par C on the activities of apoptosis markers and the levels of BDNF. The Caspase-3 (A), Caspase-9 (B), and BDNF (C) levels in brain tissue were measured by ELISA. Data were shown as mean $\pm \mathrm{SD}(\mathrm{n}=8)$. ***P $<0.00 \mathrm{I}$, $* * \mathrm{P}<0.01$, no significant difference (ns). MCAO+HPar: I00 mg/kg body weight Par $\mathrm{C}$ treatment dose; MCAO+Eda: $3 \mathrm{mg} / \mathrm{kg}$ body weight Eda treatment dose.

Abbreviations: SD, standard deviation; Par C, Parishin C; MCAO, middle cerebral artery occlusion; brain-derived neurotrophic factor (BDNF).
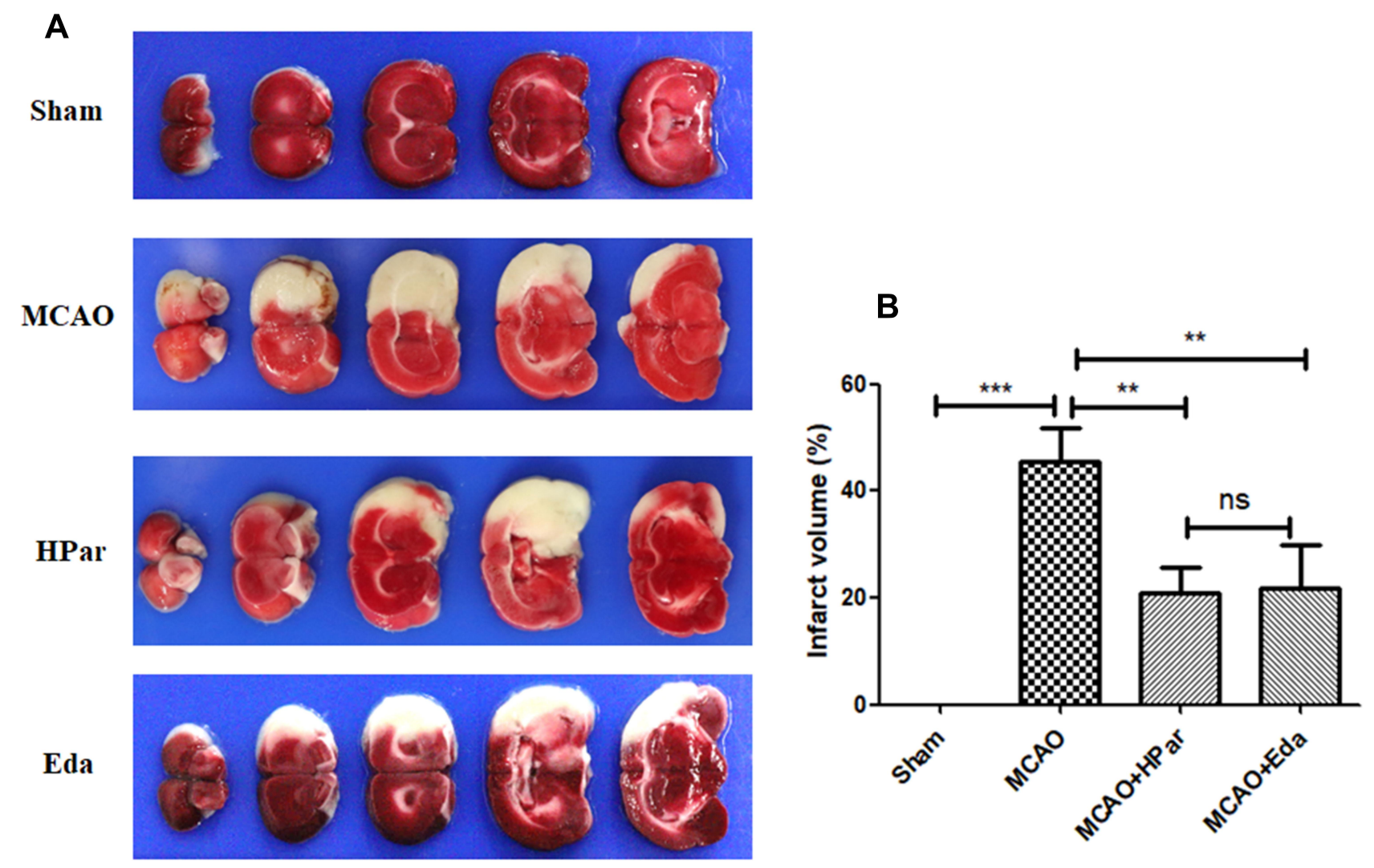

Figure 8 Effect of Par C on cerebral infarct volume after MCAO in rats. Representative micrographs of TTC staining brain sections in the four groups (A). Infarct volume percentage $(B)$. Data were shown as mean $\pm S D(n=8)$. **** $<0.001$, $* * p<0.01$, no significant difference (ns).

evidence indicated that inflammatory response could lead has been reported that cerebral ischemia-reperfusion may to extreme tissue injury, such as triggering toxic enzymes destruct cell membranes of neurons and activate microglia of brain tissue, as well as accumulation of free radical. ${ }^{32}$ It cells, resulting in further release of pro-inflammatory

Table 3 Correlation Analysis Was Performed Between the Neurological Score and Inflammatory and Oxidative Stress Parameters

\begin{tabular}{|l|l|l|l|l|l|l|l|}
\hline & TNF- $\alpha$ & IL-6 & IL-I $\beta$ & SOD & CAT & GSH-Px & MDA \\
\hline Neurological score & $0.97 I^{*}$ & $0.961^{*}$ & $0.941^{*}$ & $-0.929^{*}$ & $-0.961^{*}$ & $-0.904^{*}$ & $0.967^{*}$ \\
\hline
\end{tabular}

Notes: *Correlation is significant at $P<0.05$ level. 


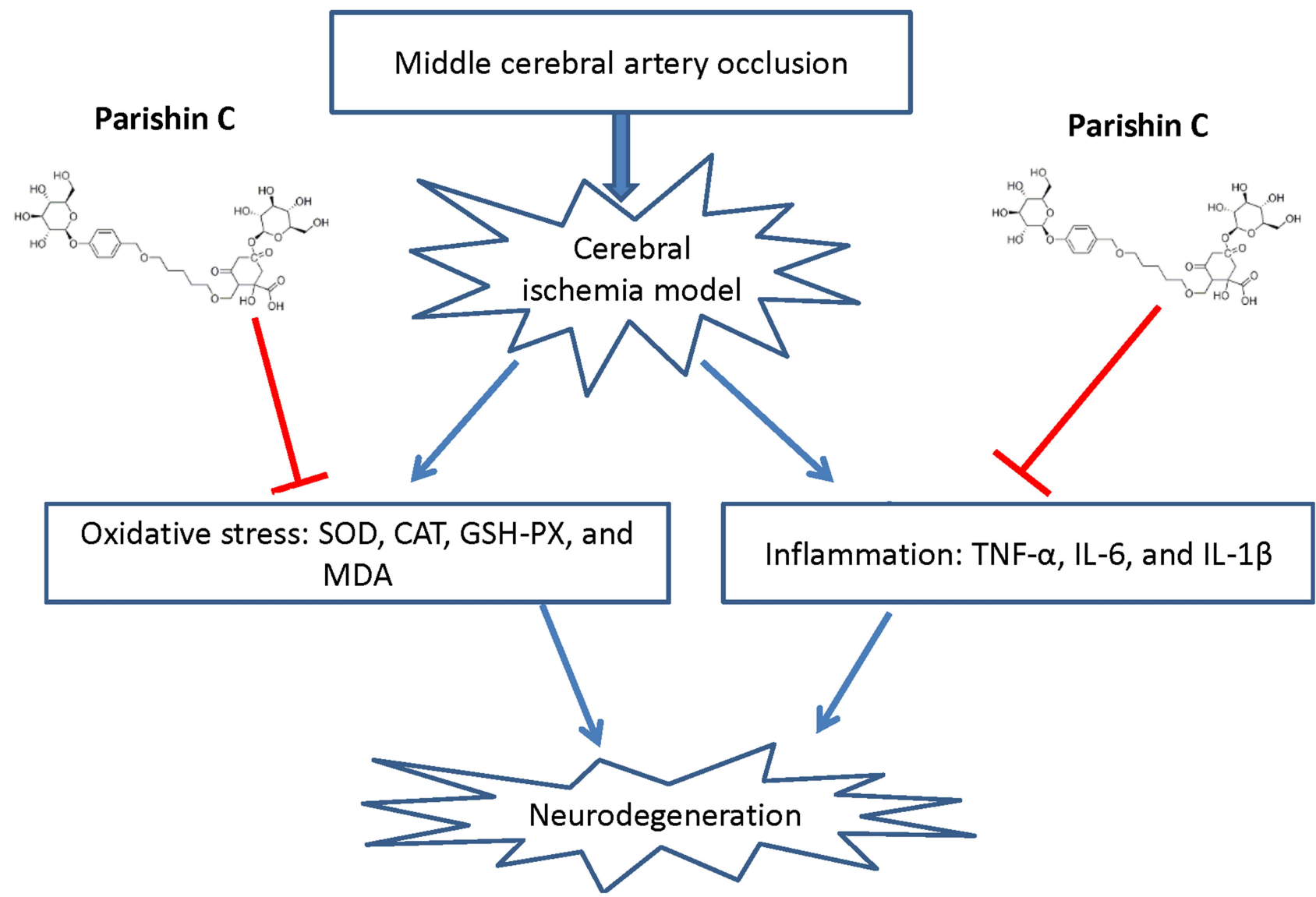

Figure 9 Schematic diagram illustrating possible neuroprotective mechanisms of Par $C$ against the MCAO-induced neurodegeneration via inhibiting oxidative stress and inflammation.

cytokines to exacerbate neuronal damage. ${ }^{33}$ Thus, suppression of inflammatory response could alleviate neuronal ischemia injury and decrease infarct size. ${ }^{34,35}$ Here we showed that Par $\mathrm{C}$ alleviated neuroinflammation as evidenced via down-regulating pro-inflammatory factors such as TNF- $\alpha$, IL-6, and IL- $1 \beta$. All of the results suggested that the neuroprotective effects of Par might be attributed to the inhibition of oxidative stress damage and neuroinflammation.

There are several limitations to the present study. For example, due to other reasons, the RT-qPCR analysis did not set different Par $\mathrm{C}$ doses, so it did not exhibit dosedependent results. Also, our study did not systematically investigate the molecular mechanism that is involved in the neuroprotective effects of Par $\mathrm{C}$ against cerebral ischemic/reperfusion injury. Although the present results demonstrated the neuroprotective effects of Par in a rat model, the clinical studies should be performed in future research.
In conclusion, our findings demonstrated that Par $\mathrm{C}$ alleviates MCAO-induced cerebral ischemia injury via inhibiting neuroinflammation and oxidative stress (Figure 9). These results implied that Par C possibly is a potential natural therapeutic drug for the prevention and treatment of ischemic stroke.

\section{Funding}

The authors received no financial support for the research, authorship, and/or publication of this article.

\section{Disclosure}

The authors declared no potential conflicts of interest with respect to the research, authorship, and/or publications of this article.

\section{References}

1. Lapi D, Colantuoni A. Remodeling of Cerebral Microcirculation after Ischemia-Reperfusion. J Vasc Res. 2015;52(1):22-31. doi:10.1159/ 000381096 
2. Mozaffarian D, Benjamin EJ, Go AS, et al. Heart disease and stroke statistics-2015 update: a report from the American Heart Association. Circulation. 2015;131:e29-322. doi:10.1161/ CIR.0000000000000152

3. Pang T, Zhao J, Zhang F, et al. Protective Effects of Yiqi Xingnao Oral Liquid on Cerebral Ischemia-Reperfusion Injury in Rats and Its Related Mechanisms. Evid Based Complement Alter Med. 2020;2020:3268047. doi:10.1155/2020/3268047

4. Herson PS, Traystman RJ. Animal models of stroke: translational potential at present and in 2050. Future Neurol. 2014;9:541-551. doi:10.2217/fnl.14.44

5. Tuttolomondo A, Di Sciacca R, Di Raimondo D, Renda C, Pinto A, Licata G. Inflammation as a therapeutic target in acute ischemic stroke treatment. Curr Top Med Chem. 2009;9:1240-1260. doi:10.2174/156802609789869619

6. Li P, Stetler RA, Leak RK, et al. Oxidative stress and DNA damage after cerebral ischemia: potential therapeutic targets to repair the genome and improve stroke recovery. Neuropharmacology. 2018;134:208-217. doi:10.1016/j.neuropharm.2017.11.011

7. Lugrin J, Rosenblatt-Velin N, Parapanov R, Liaudet L. The role of oxidative stress during inflammatory processes. Biol Chem. 2014;395:203-230.

8. Wicha P, Tocharus J, Janyou A, et al. Hexahydrocurcumin protects against cerebral ischemia/reperfusion injury, attenuates inflammation, and improves antioxidant defenses in a rat stroke model. PLoS One. 2017;12(12):e0189211. doi:10.1371/journal.pone.0189211

9. Zhan HD, Zhou HY, Sui YP, et al. The rhizome of Gastrodia elata Blume - An ethnopharmacological review. $J$ Ethnopharmacol. 2016;189:361-385. doi:10.1016/j.jep.2016.06.057

10. Shin EJ, Whang WK, Kim S, et al. Parishin C attenuates phencyclidine-induced schizophrenia-like psychosis in mice: involvements of 5-HT1A receptor. J Pharmacol Sci. 2010;113:404-408. doi:10.1254/jphs.10040SC

11. Liu Z, Wang W, Feng N, Wang L, Shi J, Wang X. Parishin C's prevention of $A \beta$ 1-42-induced inhibition of long-term potentiation is related to NMDA receptors. Acta Pharm Sin B. 2016;6:189-197. doi:10.1016/j.apsb.2016.03.009

12. Lin Y, Sun Y, Weng Y, Matsuura A, Xiang L, Qi J. Parishin from Gastrodia elata Extends the Lifespan of Yeast via Regulation of Sir2/ Uth1/TOR Signaling Pathway. Oxid Med Cell Longev. 2016;2016:4074690. doi:10.1155/2016/4074690

13. Hua K, Sheng X, Li TT, et al. The edaravone and 3-n-butylphthalide ring-opening derivative $10 \mathrm{~b}$ effectively attenuates cerebral ischemia injury in rats. Acta Pharmacol Sin. 2015;36:917-927. doi:10.1038/ aps.2015.31

14. Xue J, Zhang X, Zhang C, et al. Protective effect of Naoxintong against cerebral ischemia reperfusion injury in mice. $J \quad$ Ethnopharmacol. 2016;182:181-189. doi:10.1016/j. jep.2016.02.022

15. Viswanatha GL, Venkataranganna MV, Prasad NBL, Shylaja H. Achyranthes aspera Linn. alleviates cerebral ischemia-reperfusioninduced neurocognitive, biochemical, morphological and histological alterations in Wistar rats. J Ethnopharmacol. 2019;228:58-69. doi:10.1016/j.jep.2018.09.018

16. Durukan A, Tatlisumak T. Acute ischemic stroke: overview of major experimental rodent models, pathophysiology, and therapy of focal cerebral ischemia. Pharmacol Biochem Behav. 2007;87:179-197. doi:10.1016/j.pbb.2007.04.015

17. Speetzen LJ, Endres M, Kunz A. Bilateral common carotid artery occlusion as an adequate preconditioning stimulus to induce early ischemic tolerance to focal cerebral ischemia. J Visualized Exp. 2013; e4387. doi:10.3791/4387
18. Cheng CY, Su SY, Tang NY, Ho TY, Chiang SY, Hsieh CL. Ferulic acid provides neuroprotection against oxidative stress-related apoptosis after cerebral ischemia/reperfusion injury by inhibiting ICAM-1 mRNA expression in rats. Brain Res. 2008;1209:136-150. doi:10.1016/j.brainres.2008.02.090

19. Valko M, Leibfritz D, Moncol J, Cronin MT, Mazur M, Telser J. Free radicals and antioxidants in normal physiological functions and human disease. Int J Biochem Cell Biol. 2007;39:44-84.

20. Shirley R, Ord EN, Work LM. Oxidative Stress and the Use of Antioxidants in Stroke. Antioxidants. 2014;3:472-501. doi:10.3390/ antiox3030472

21. Sommer CJ. Ischemic stroke: experimental models and reality. Acta Neuropathol. 2017;133:245-261. doi:10.1007/s00401-017-1667-0

22. Yuan Y, Men W, Shan X, et al. Baicalein exerts neuroprotective effect against ischaemic/reperfusion injury via alteration of NF-kB and LOX and AMPK/Nrf2 pathway. Inflammopharmacology. 2020;28 (5):1327-1341. doi:10.1007/s10787-020-00714-6

23. He J, Wu H, Zhou Y, Zheng C. Tomentosin inhibit cerebral ischemia/ reperfusion induced inflammatory response via TLR4/NLRP3 signalling pathway - in vivo and in vitro studies. Biomed Pharmacother. 2020;131:110697. doi:10.1016/j.biopha.2020.110697

24. Zhang R, Liu C, Li Y, Chen L, Xiang J. Tenacissoside H Promotes Neurological Recovery of Cerebral Ischemia-reperfusion Injury in Mice by Modulating Inflammation and Oxidative stress via TrkB Pathway. Clin Exp Pharmacol Physiol. 2021;48:757-769. doi:10.1111/1440-1681.13398

25. Ren ZL, Zuo PP. Neural regeneration: role of traditional Chinese medicine in neurological diseases treatment. $J$ Pharmacol Sci. 2012;120:139-145. doi:10.1254/jphs.12R06CP

26. Qiao $\mathrm{Z}, \mathrm{Xu}$ Y. SALVIANOLIC ACID B ALLEVIATING MYOCARDIUM INJURY IN ISCHEMIA REPERFUSION RATS. African $j$ Traditional Complement Alter Med. 2016;13:157-161. doi:10.21010/ajtcam.v13i4.20

27. Chen S, Sun M, Zhao X, et al. Neuroprotection of hydroxysafflor yellow A in experimental cerebral ischemia/reperfusion injury via metabolic inhibition of phenylalanine and mitochondrial biogenesis. Mol Med Rep. 2019;19:3009-3020. doi:10.3892/mmr.2019.9959

28. Rodrigo R, Fernández-Gajardo R, Gutiérrez R, et al. Oxidative stress and pathophysiology of ischemic stroke: novel therapeutic opportunities. CNS Neurol Disord Drug Targets. 2013;12:698-714. doi:10.2174/1871527311312050015

29. Allen CL, Bayraktutan U. Oxidative stress and its role in the pathogenesis of ischaemic stroke. Int $j$ Stroke. 2009;4:461-470. doi:10.1111/j.1747-4949.2009.00387.x

30. Jin R, Yang G, Li G. Inflammatory mechanisms in ischemic stroke: role of inflammatory cells. J Leukoc Biol. 2010;87:779-789. doi:10.1189/jlb.1109766

31. Xing C, Arai K, Lo EH, Hommel M. Pathophysiologic cascades in ischemic stroke. Int $j$ Stroke. 2012;7:378-385. doi:10.1111/j.17474949.2012.00839.x

32. Gill R, Tsung A, Billiar T. Linking oxidative stress to inflammation: toll-like receptors. Free Radic Biol Med. 2010;48:1121-1132. doi:10.1016/j.freeradbiomed.2010.01.006

33. Kaushal V, Schlichter LC. Mechanisms of microglia-mediated neurotoxicity in a new model of the stroke penumbra. $J$ Neurosci. 2008;28:2221-2230. doi:10.1523/JNEUROSCI.5643-07.2008

34. Siniscalchi A, Gallelli L, Malferrari G, et al. Cerebral stroke injury: the role of cytokines and brain inflammation. J Basic Clin Physiol Pharmacol. 2014;25:131-137. doi:10.1515/jbcpp-2013-0121

35. Zhao TZ, Ding Q, Hu J, He SM, Shi F, Ma LT. GPER expressed on microglia mediates the anti-inflammatory effect of estradiol in ischemic stroke. Brain Behav. 2016;6:e00449. doi:10.1002/brb3.449 


\section{Publish your work in this journal}

Neuropsychiatric Disease and Treatment is an international, peerreviewed journal of clinical therapeutics and pharmacology focusing on concise rapid reporting of clinical or pre-clinical studies on a range of neuropsychiatric and neurological disorders. This journal is indexed on PubMed Central, the 'PsycINFO' database and CAS, and is the official journal of The International Neuropsychiatric Association (INA). The manuscript management system is completely online and includes a very quick and fair peer-review system, which is all easy to use. Visit http://www.dovepress.com/testimonials.php to read real quotes from published authors. 